\title{
Das Homunculus-Motiv bei Paracelsus und Goethe
}

\author{
Von Dieter Kerner
}
«... daß der Homunculus von derselben Hand geschaffen sei wie wir...»
L. STERNE, Tristram Shandy

Die Legende von der Erschaffung eines künstlichen Menschen gründet sich nicht allein auf das Gedankengut des Mittelalters und der Frühromantik, sondern basiert auf einer jahrtausendealten religionsgeschichtlichen Tradition.

Während nämlich die Genesis nur die Verleihung der Seele, des Lebensodems (1. Mos. 2, 7) durch Gott an den aus Erde erschaffenen Adam kennt, fehlen in der frühen jüdischen Überlieferung nicht Äußerungen über einen tellurischen Erdgeist, der dem ersten Menschen zusätzlich innegewohnt haben soll. Diesen zu wecken und kraft magischer Evokation zu einer untergeordneten Seinsform zu beleben, war Gegenstand zahlreicher kabbalistischer Praktiken, zumal eine Bibelstelle wie «Die Erde bringe lebendige Wesen hervor» (1. Mos. 1, 24) als indirekte Bestätigung für die Möglichkeit einer geistigen Emanation aus grobstofflichem Material ohne « ein von oben eingeblasenes Pneuma» aufgefaßt werden konnte. Folglich wird Adam stellenweise in einem gewissen Stadium seiner Entstehung aus dem Rohzustand als Golen bezeichnet; diese Vorstellung ist um so einleuchtender, da er ja von Erde genommen ist und einst in diesen Urzustand zurückkehrt.

«Golem» stellt ein hebräisches Wort dar, das in der Bibel nur an einer Stelle, und zwar im Psalm 139,16, vorkommt:

«Gesehen haben Deine Augen meine ungeformten Glieder», wobei dem Worte Golem unmißverständlich das Prädikat des Ungestalteten, Formlosen eigen ist, was auch durch den übrigen Sinn dieser Psalmworte klar wird: «... Obgleich ich im Verborgenen geschaffen ward, gewirkt in Erdentiefen.»Den von dem Anhauch Gottes noch nicht betroffenen Adam hat man in dieser Version als Golem bezeichnet, ehe die Seele in ihn geworfen war und bevor er sprach, befand er sich auf einer niederen Stufe. Die Nachbildung eines solchen Wesens zu versuchen, das sich im Vergleich zum Su- 
perlativ des fertigen Menschen nur wie ein Komparativ in der Rangordnung belebter Individuen ausnahm und für dessen Existenz nicht Gott, sondern in eigenmächtiger Selbstüberheblichkeit der Mensch die letzte Verantwortung zu übernehmen bereit war - die Nachbildung einer solchen Figur hat bereits im Altertum nicht wenige Denker beschäftigt. Daß mithin der Gerechte und Schriftkundige, wenn er nur die 72 Buchstaben des Gottesnamens (21) gemäß den Anweisungen des kabbalistischen Buches Jezira (7) richtig kombinierte, kraft seines Glaubens Berge versetzen und ein künstlich Gebilde zu produzieren imstande schien, liegt auf der Hand. Und warum sollte dann einem Sterblichen solcherlei nicht möglich sein ? Heißt es doch bei Jesaja $(59,2)$ : «Denn eure Sünden machen eine Scheidung zwischen euch und eurem Gott.» - Eine treffende Schilderung dieser ganzen Prozedur lieferte ЈАков Griмm (20) in der romantischen Zeitung für Einsiedler aus dem Jahre 1808:

«Die polnischen Juden machen nach gewissen gesprochenen Gebeten und gehaltenen Fasttagen die Gestalt eines Menschen aus Ton oder Leimen, und wenn sie den wundertätigen Gottesnamen darüber sprechen, so muß er lebendig werden. Reden kann er zwar nicht, versteht aber ziemlich, was man spricht oder befiehlt. Sie heißen ihn Golem und brauchen ihn zu einem Aufwärter, allerlei Hausarbeit zu verrichten. Allein er darf nimmer aus dem Hause gehen. An seiner Stirn steht geschrieben emeth (Wahrheit), er nimmt aber täglich zu und wird leicht größer und stärker als alle Hausgenossen, so klein er anfangs gewesen ist. Daher sie aus Furcht vor ihm den ersten Buchstaben auslöschen, so daß nichts bleibt als meth (er ist tot), worauf er zusammenfällt und wieder in Ton aufgelöst wird. Einem ist sein Golem aber einmal so hoch geworden und hat ihn aus Sorglosigkeit immer wachsen lassen, daß er ihm nicht mehr an die Stirn hat reichen können. Da hat er aus der großen Angst den Knecht geheißen, ihm die Stiefel auszuziehen, in der Meinung, daß er ihm beim Bücken an die Stirn reichen könne. Dies ist auch geschehen und der erste Buchstabe glücklich ausgetan worden, allein die ganze Leimlast fiel auf den Juden und erdrückte ihn.»

Obige Quelle, die man leicht als eine dichterische Erfindung ansehen könnte, stützt sich letzten Endes auf einen alten talmudischen (1) Bericht: «Rabha nämlich schuf einen Menschen (Mann) und schickte ihn zu Rabbi Zera. Der sprach mit ihm, und er gab keine Antwort. Da sagte er: ,Du stammst wohl von den Magiern; kehre zu deinem Staub zurück!'» In erweiterter Form hat dieser Traktat später Eingang in das kabbalistische Buch Bahir (6) gefunden, was seine Bedeutung noch unterstreicht. Rabha vermochte zwar einen Mann zu schaffen, der bis zu Rabbi Zera gehen konnte, die Sprache aber konnte er ihm nicht verleihen, und an dem stummen Wesen erkannte Rabbi Zera die Natur seines Gegenübers. Der Golem tritt dann später als Bezeichnung der durch das magische Vermögen des Men- 
schen erschaffenen menschenähnlichen Gestalt vom Ende des 12.Jahrhunderts an mehrfach in den Texten auf, und wenn GoEthe die Rolle des Homunculus von einem Bauchredner (zu Eckermann, 20. Dezember 1829) interpretiert wissen wollte, so spielten die alten Gedankengänge von der Stimmlosigkeit des künstlichen Menschen sicher eine wesentliche Rolle. Allerdings hat im Laufe der Zeit die Qualität der Golem-Vorstellung einen nicht unerheblichen Wandel erfahren. Nach dem Sohar und anderen kabbalistischen Werken wurde nämlich die Schaffung des künstlichen Menschen immer mehr von einer erlaubten Tat des Frommen und Gottesfürchtigen in einen Akt Schwarzer Magie transferiert - Magie erscheint nun als ein erst im Fall Adams sich eröffnendes Verfahren zur Erkenntnis des «eritis sicut deus scientes bonum et malum ", wovon sich wiederum die Verfallenheit des Menschen an den Tod herleitet. Die Blätter vom Baume der Erkenntnis, mit denen Adam seine Blöße verhüllt, werden zum zentralen Symbol des eigentlichen magischen Wissens (20). Die Golem-Genese, einst das Schöpfungsergebnis kindlich-naiver Glaubenskraft mit spielerischem Unterton, erhielt jetzt eine Wendung zum Prometheisch-Luziferischen hin. Sie wurde zum Zeichen des Eingeweihten, des Initiierten, wobei jedoch nicht zu vergessen ist, daß auch dieser künstliche Golem jederzeit durch die Wegnahme der belebenden Buchstabenkombination an der Stirn wieder zu Staub zerfällt.

Im 12. Jahrhundert erreichte dann die Flut der «Rezepte» über die Details der Golem-Schöpfung auf «mystischer Erfahrung» ihren Höhepunkt. Aber in keiner Quelle tritt bis dahin ein auf solche Weise erschaffener Golem ins wirkliche Leben ein, er übernimmt auch nirgendwo eine Funktion. Die formellste und älteste Vorschrift findet sich bei Eleasar aus Worms (1160-1230) am Ende seines Jezira-Kommentars mit den dazugehörigen Tafeln der Kombinationen des Alphabets. Das Wesentliche der Anweisung besteht darin, daß die sich zum Golem-Ritual verbindenden zwei oder drei Adepten unbearbeitete Bergerde nehmen, die sie in fließendem Wasser kneten und daraus die Figur formen. Über dieselbe sollen sie dann die aus den «Pforten» des Buches Jezira sich ergebenden Kombinationen des Alphabets sprechen - und über jedes Körperglied denjenigen Konsonanten, der ihm im Buch Jezira zugeordnet ist -, welche bei ihm die immerhin stattliche Anzahl von 221 Variationen erreichen. Eine Umkehrung dieser Ordnungen begleitet die Rückverwandlung des zum Leben erwachten Golems in Staub. Andere Schöpfungsvorschriften, die zum Teil einen magischen Kreis als obligat ansehen, tragen sogar ekstatischen Charakter, oft 
wird der dann unvermeidliche Mißerfolg aller dieser Praktiken der Unzulänglichkeit der schriftlich überlieferten Gebrauchsanweisung zur Last gelegt. Dabei war der Akt als solcher, begleitet von einer besonderen Atemtechnik und entsprechenden Kopf- oder Gliederbewegungen, nicht ungefährlich. Irrtümer konnten sich gegen den Schöpfer selbst richten und diesen wie einen Golem vernichten, obwohl der eigentliche Golem, da ohne Sprache, auch ohne Seele gedacht war (20). Zu Anfang des 14. Jahrhunderts tauchten dann Golem-Rezepte auf, die bei der Herstellung ein Gefäß mit einbezogen - die Überleitung zum paracelsischen «Kolben» und zur «Phiole» bei GoEтhe schien hergestellt, entsprechend dem Goethe-Wort:

$$
\begin{aligned}
& \text { «Natürlichem genügt das Weltall kaum; } \\
& \text { Was künstlich ist, verlangt geschloßnen Raum.» }
\end{aligned}
$$

Die «Chymische Anthropogenesis» bei Paracelsus wird am ausführlichsten im 1. Buch der neun Teile De Natura rerum beschrieben:

«Nun ist aber auch die generation der homunculi in keinen weg zu vergessen. dan etwas ist daran, wiewol solches bisher in großer heimlikeit und gar verborgen ist gehalten worden und nit ein kleiner zweifel und frag under etlichen der alten philosophis gewesen, ob auch der natur und kunst möglich sei, das ein mensch außerthalben weiblichen leibs und einer natürlichen muter möge geboren werden? darauf gib ich die antwort, das es der kunst spagirica und der natur in keinem weg zuwider, sonder gar wol möglich sei. wie aber solches zugang und geschehen möge, ist nun sein proce $\beta$ also, nemlich das der sperma eines mans in verschloßnen cucurbiten (Kolben) per se mit der höchsten putrefaction, ventre equino (gärender Pferdemist) putreficirt werde auf 40 tag oder so lang bis er lebendig werde und sich beweg und rege, welches leichtlich zu sehen ist. nach solcher zeit wird es etlicher maßen einem menschen gleich sehen, doch durchsichtig on ein corpus. so er nun nach disem teglich mit dem arcano sanguinis humani gar weislich gespeiset und erneret wird bis auf 40 wochen und in steter gleicher werme ventris equini erhalten, wird ein recht lebendig menschlich kint daraus mit allen glitmaßen wie ein ander kint, das von einem weib geboren wird, doch vil kleiner. dasselbig wir ein homunculum nennen und sol hernach nit anders als ein anders kint mit großem fleiß und sorg auferzogen werden, bis es zu seinen tagen und verstant kompt. das ist nun der aller höchsten und größesten heimlikeiten eine, die got den tötlichen und sündigen menschen hat wissen lassen. dan es ist ein mirakel und magnale dei und ein geheimnis uber alle geheimnus ...» (13)

Ob der Verfasser, wie SudHoff sogar im Vorwort zum 11. Band seiner Paracelsus-Gesamtausgabe glaubte, eine solche Homunculus-Konzeption nur als larvierten Ulk an den Leser brachte, kann man kaum annehmen unter Berücksichtigung der Tatsache, daß Paracelsus dieses Problem mehrfach in seinem Gesamtwerk angeschnitten und mit hohem Ernst kommentiert hat. Auch die Zahl 40, womit er sonst gerne jenen Wochenabschnitt 
bezeichnet, den das Kind zur Reifung im Mutterleib benötigt, ist ein typisch paracelsischer Terminus technicus. In der Schrift Von dem Honig (11) streift Paracelsus dasselbe Thema mit den Worten "gleich wie in einem roßmist digerirt wird homunculus», in den Deutschen Originalfragmenten $z u$ den fünf Büchern «De vita longa» (12) spricht er wieder vom «homunculus ... dan generatio homunculi ist durch große digestion im ventre equino gemacht, und sicht dem gleich, von dem es komt, im fleisch und blut der merern und wenigern glidern ...» - eigentlich eine Wiederholung seiner an anderer Stelle erfolgten Beschreibung des synthetischen Menschleins, das er im Liber Azoth (14) durch Schwarzweißmalerei im Sinne von Gut und Böse noch mit den Prädikaten «aquastrisch» und «cagastrisch» belegt. Endlich wird im wohl pseudoparacelsischen Traktat De Pestilitate (15) das gewonnene Erfahrungsgut vom banalen Hexenzauber überwuchert, es erscheint sogar ein homunculus cereus, ein Abbild aus Wachs, das man beliebig modellieren kann, um durch einen «vergifteten Spiegel» dann allen bösen Wünschen, womit man den persönlichen Widersacher bedenkt, magischen Nachdruck zu verleihen, so daß der homunculus cereus quasi als Doppelgänger des Behexten wirkt. Dennoch hat Paracelsus sicher an die Wirksamkeit derartiger künstlicher Gebilde geglaubt, schreibt er doch im 12. Kapitel seines Liber de Imaginibus (16) davon, «wie und in was gestalt die homunculi und bilder gemacht und zugericht werden, in denen eine heimliche menschheit spiritualischer weise verborgen ligt. und darumb werden dise bilder homunculi genant, das sie sollen alle gestalt und glitmaß haben wie ein mensch, iedoch nicht als groß als ein mensch, sonder als klein mans machen kan.» Der Hohenheimer liefert dann ein eingehendes Modulationsverfahren aus Erde, Wachs und Metall mit, ansonsten müssen «dise homunculi und bilder gemacht werden ... nach dem inneren wesen des menschen. die selbigen influenzen müssen aus dem innern wesen gên in das eußer wesen, das ist aus dem menschen in ein homunculum.»

Wer dies liest, könnte zu der Vorstellung gelangen, Paracelsus habe die Konstruktion des Homunculus lediglich im talismanischen Sinn verstanden nach der Regel «wilt du dein feint binden, so bint sein bilt», aber der Homunculus ist für ihn mehr als nur wachsgewordene Gemme, er ist ein individuum per se. Das erweist mit letzter Deutlichkeit die «Philosophia magna », worin er diesem Problem ein besonderes Kapitel, Liber de homunculis (17), gewidmet hat. Daß dies im Zenit seines Schaffens geschah, wirft ein bezeichnendes Licht auf die Intensität des inneren Anliegens. Leider ist der Traktat unvollständig, an vielen Stellen bricht die Handschrift jäh ab, 
und es klaffen erhebliche Lücken. Soviel wird aber immerhin zur Genüge deutlich, daß Paracelsus eine scharfe Trennung zwischen Beseeltem und Unbeseeltem, zwischen den Menschen einerseits und den Homunculis oder Monstris andrerseits durchführt: «das ungesêlt aber ist nichts», wie eine taube $\mathrm{Nu} \beta$, «dan der mensch sol ein menschen geberen und nicht ein vich.» Weit holt der Verfasser aus, um dem Leser im einzelnen seine affektgeladenen Gedanken verständlich zu machen, um die Herkunft dieser «Mißgewächse » zu erklären, für die er in erster Linie jede Art widernatürlicher Sexualität, das «satanische Wesen» verantwortlich macht, «denn das wissen aber, was nicht in der matrix (Gebärmutter) empfangen ist durch die natürlichen werken, das ist alles zum monstro und mißgewechs geneigt ... ist aber kein samen mer, sonder ein materia homunculi.» In diese Erwägungen spielen sicherlich auch kabbalistische Gedanken mit herein, wonach die Entstehung von Dämonen durch Onanie erfolgt (20). «aber weiter so wissen auch von dem spermate der tieren, das sie oftmals sodomiren, die selbigen an mannen stat gebrauchen. daraus dan sonderlich monstra geboren werden, die da in der form und gestalt gleich sehen den selbigen tieren.» Solche Monstra «aus sodomitischer Ordnung» sind weder Mensch noch Tier, «sonder in alle weg ein mißgewechs, das erschrecklich ist ... anzusehen.» Selbst im Magen können sie sich unter entsprechenden Bedingungen nach seiner Vorstellung entwickeln. «... dadurch sie wol mögen verstên, so solch ding nicht nach der natur geschehen und ir ordnung, das ist nichts dan sodomiten arbeit, aus welchen solche ungebürlich ding wachsen und entspringen, und in so vil weg und form gebraucht, das die monstra und homunculi nicht all mögen beschriben werden, sonder nach der kürzi erzelet und fürgehalten, solch laster zu vermeiden und die zu erkennen, so damit umbgehent, auch was jungfrauschaft sei, was frau sei, und was die nit seient, das ist, was sodomiten seind ...» Freilich faßt Paracelsus diesen letzteren Wortbegriff nicht so eng, wie wir es heute gewohnt sind, so wird für ihn auch aus der «Sodomie» zwischen Esel und Roß das Maultier, aus der Kreuzung von Hahn und Kröte der schon bei JesaJA $(59,5)$ erwähnte Basilisk, die Meerjungfrau ist das Produkt von Frau und Fisch (18). Eines aber haben diese heterogen entstandenen, aus Fehlbefruchtung erwachsenen «sodomitischen Monstra» miteinander gemein: sie sind seelenlos, «on sêl»!

Der von Paracelsus im 1. Buch De Natura rerum (13) vorgetragene Gedanke «Es ist auch zu wissen, das also menschen mögen geboren werden one natürliche veter und mütter» ist bis in das Gesamtwerk GoEthES zu verfolgen, der laut eigener Angabe (Dichtung und Wahrheit, Buch 8) die 
paracelsischen Schriften selbst studierte. Um so faszinierender mußte auf den Olympier Goethe das damals verfemte Wissen des reisenden Arztes gewirkt haben, stand doch über ihn in dem Buch von Johann Georg Zimmermann Von der Erfahrung der Arzneykunst (23) geschrieben: «Paracelsus ... erfrechte sich vollends zu lügen, er könne vermittelst der Chemie ein wahrhaft lebendes Kind hervorbringen.» GoEthes «Mensch aus der Retorte», der Homunculus (Faust, II, Akt 2), durchläuft den analogen Entwicklungsgang wie bei Paracelsus (5), er leuchtet, ja schwebt sogar und wird bezeichnenderweise gleich durch die Deutung eines typisch phallischen Traumes (Leda und der Schwan) mit seinen geistigen Vätern Faust und Mephisto in Zusammenhang gebracht. Ansonsten ist der Menschenstoff wie üblich «gemischt» und «komponiert», im Kolben "verlutiert» (d.h. eingeschlossen; was sich übrigens wieder vom Worte «Lehm», der Ursubstanz jeder schöpferischen Materialisation, herleitet!), kohobiert (doppelt destilliert; synonym für «Putrefaction» mittels feuchter Wärme), organisiert und kristallisiert. Selbst im paracelsischen Sinne «durchsichtig» erscheint dieser Homunculus «im Glas», der Prosaentwurf vom 17. Dezember 1826 sah außerdem die Zersprengung des hellen Kolbens durch den kleinen «Geist in der Flasche» vor (5), auf Grund anderer Erwägungen sollte er als Kalendermännlein (4) in Aktion treten unter Bezugnahme auf besondere historische Ereignisse seit der Erschaffung Adams.

Wichtig ist der Umstand, daß Goethes Homunculus a priori in die Reihe der höheren Geister aufgenommen erscheint und zu Mephisto in einem Vetternverhältnis steht. Goethe hat dann gegenüber Eckermann (3) am 16. Dezember 1829 auch keinen Zweifel an der dämonischen Wesensart dieses künstlichen Menschen aufkommen lassen. Erst dadurch wird der Homunculus ja auch in die Lage versetzt, während der Klassischen Walpurgisnacht die Verbindung zu den Gefilden Griechenlands herzustellen, bevor er zuletzt selbst den Elementen zum Opfer fällt.

Im Plan vom 9. November 1826 notierte Goethe: «Wagners Laboratorium. Er sucht ein chemisch Menschlein hervorzubringen»(5). Wie zufällig hatte hier der Dichter, wenn auch auf andere Weise, die Begegnung des vergänglichen Individuums mit dem Numinosen wiederholt - das Gespräch des Dr. Faustus mit dem Erdgeist im 1.Teil der Tragödie war letztlich nur eine Umkehrung dieser Szene, und jedesmal kam der Adept Wagner ins Bild - als der Erdgeist verglühte und «sein » Homunculus erglühte! Daß sich das Divine immer wieder als strahlende Energie kundgibt - nie ist dies vorher, mit Ausnahme der Bibel, derart deutlich zum Ausdruck gebracht 
worden, nicht einmal bei Paracelsus. Dafür aber hatte der Hohenheimer alle nur denkbaren Entwicklungsmöglichkeiten für solcherlei Kunstprodukte offen gelassen, «dan aus disen homunculis, so sie zu menlichem alter komen, werden risen, zwerglen und ander dergleichen große wunderleut... darumb das sie mit iren kreften und taten nit menschen sondern sich geistern vergleichen» (13). Aber es kann ihnen ergehen wie den Monstris und wie dem Goetheschen Homunculus: Ihnen ist ein früher Tod fast sicher, « und ie wunderbarer und schröklicher die seind, ie schneller und ehe der tot auf sie folget» (13).

Überprüft man das, was Paracelsus zu diesem Thema gesagt hat, und vergleicht man es mit der Deutung eines Goethe, so ergeben sich doch einige neue Gesichtspunkte insofern, als entgegen der alten jüdischen Vorstellung jetzt nicht mehr die Elemente Wasser und Erde als eigentliche Urstoffe anzusprechen sind, auch ist die Zeitangabe von 40 Tagen, die zwischen der Einschließung des Spermas in die Retorte und der Bildung des Homunculus vergehen, neu. Weiter fällt auf, daß bei Paracelsus mit den «Homunculis» sogar die golemähnlichen Figuren aus Wachs, Lehm oder Pech gemeint sind, die beim Schadenzauber der Schwarzen Magie benutzt werden (20). Schließlich reift im 17. Jahrhundert - ganz entgegen der vorherigen Tradition - der Homunculus zum dämonischen Diener des Menschen heran, und indem er sich immer mehr verselbständigt, wird er zum «fabelhaften Wesen» der Literatur, auf welches die Dichter der Romantik (Wieland, Sterne, Lichtenberg, Rousseau, Arnim, Hoffmann) mit Vorliebe zurückgriffen. Im «Zauberlehrling» GoEтHEs findet außerdem eine Wendung des Homunculus-Bildes hin zum Gefährlichen, Bösartigen, statt, welche auch für die hebräische Literatur des 18. Jahrhunderts richtungweisend werden sollte. Jetzt verfügt der Golem plötzlich über ungeheure Kräfte, wächst über alles Maß, zerstört gar die Welt und richtet großes Unglück an. Das Unheimliche, von dem die alten Golem-Vorstellungen überhaupt nichts zu wissen schienen, begleitet nun die Gestalt (20). Parallel mit der fortschreitenden Entwicklung der Technik projizierte das menschliche Unterbewußtsein alle destruktiven Kräfte des Maschinenzeitalters in die GolemFigur, welche sich dadurch zum merkwürdigen Zwillingswesen des Massenmenschen von heute, zum Roboter, wandelte.

Wie sehr die Gestalt des Homunculus ins Visionär-Schreckhafte verkehrt wird, unterstreicht die Sage vom «Hohen Rabbi Löw» aus Prag (etwa 1520-1609), in ihrer weitverbreiteten Lesart aus dem Anfang des 19. Jahrhunderts (20) stammend: 
Hiernach soll Rabbi Löw einen Golem gemacht haben, der zwar die ganze Woche über seinem Herrn bei verschiedenen Arbeiten diente; weil aber alle Kreaturen am Sabbath ruhen, habe der Rabbi vor Eingang des Sabbath den Golem jedesmal durch Wegnahme des belebenden Gottesnamens an der Stirn wieder zu Lehm verwandelt. Einmal jedoch vergaß der Rabbi, den Schem zu entfernen. Die Gemeinde war schon am Freitagabend zum Gottesdienst in der Synagoge versammelt, als der Golem mit ungeheurer Kraft zu toben begann, an den Häusern rüttelte und alles zu vernichten drohte. Rabbi Löw wurde herbeigerufen; die Abenddämmerung war noch nicht vorüber, und der Sabbath hatte noch nicht eigentlich begonnen*. Da stürzte er sich dem rasenden Golem entgegen und entriß ihm den Schem, und der Golem zerfiel in Erde. Der Rabbi aber habe den Golem nicht wieder zum Leben erweckt und seine Reste auf dem Dachboden der uralten Synagoge begraben, wo sie jetzt noch liegen. Aber einer der bedeutendsten Nachfolger des Rabbi Löw, Rabbi Ezechiel Landau, der einmal nach vielem Fasten heraufgestiegen sei, um die Reste des Golem zu sehen, habe für alle künftigen Geschlechter verboten, daß ein Sterblicher noch einmal diesen Versuch unternehme, in jene Dachkammer hinaufzusteigen (nach 20).

Obige Erzählung, durch viele Varianten im späteren 19.Jahrhundert umgestaltet, trotz der belletristischen Verarbeitung jedoch fest mit dem GolemStoff verflochten, wirkt noch bis in den Roman von Gustav Meyrink, betitelt Der Golem, fort, welcher im Jahre 1916 gedruckt wurde (9). Nach dieser Interpretation ist der Golem eine ahasverische Erscheinung, die alle dreiunddreißig Jahre am Fenster eines Zimmers ohne Zugang im Prager Getto erscheint und ein seltsam unerlöstes Schattendasein führt. Daß ein solches Buch ausgerechnet in einer Zeit auf den Markt kam, in der sich die entfesselten Kräfte der Technik wie ein Golem wider ihren eigenen Schöpfer, den Menschen, kehrten, ist bezeichnend. Auch der deutsche Film unter der künstlerischen Leitung von Paul Wegener nahm sich des Themas (1915 und 1920) an. Psychologisch ähnlich gelagert, nur kitschiger, wirkte der etwa gleichzeitig entstandene sechsteilige Reißer Homunculus, dessen Publikumserfolg außerordentlich war. Dabei verdient hervorgehoben zu werden, daß die kulturgeschichtlich keineswegs immer firmen Regisseure insofern ins Schwarze trafen, als sie den Homunculus im Sinne der zum Eigenbewußtsein gelangten Golem-Figur interpretierten. Schaudernd wendet sich die Umgebung von ihm ab: «Aber das ist ja Homunculus, der Mensch ohne Seele, ein Handlanger des Teufels - ein Ungeheuer!» (8). Zum rachsüchtigen Diktator geworden, entfesselt er sogar die globale Auseinandersetzung. Ein Blitzstrahl scheint vonnöten, ihn zu zerschmettern. Homunculus wie der Golem finden, vernichtungswürdig geworden, ihr gewaltsames

* Nach jüdischer Zeitrechnung beginnt der neue Tag stets schon abends gegen 18.00 Uhr nach dem Wort der Schrift «Da ward aus Abend und Morgen der erste Tag» (1. Mos. 1,5). 
Ende, das mit der Unnatur ihrer Entstehung übereinstimmt (8). Daß es sich dabei eigentlich bloß um eine Neuauflage des immer gleichbleibenden Homunculus-Stoffes handelte, wie sie auch Jacques OfFenbach in seiner Oper Hoffmanns Erzählungen (1880) in den Gestalten des Monstrums Kleinzack und der Automatenpuppe Olympia auf die Bühne brachte, sei nur am Rande erwähnt.

Weder Paracelsus noch Goethe haben also das Homunculus-Motiv inauguriert, sie stellen vielmehr Abschnitte in einer langen Entwicklungsreihe dar, die bis in die Gegenwart reicht. Dabei muß betont werden, daß die Entstehung eines künstlichen Menschengebildes wie auch das $\mathrm{Zu}-$ standekommen transzendentaler metaphysischer Erscheinungen immer mit Lichtphänomenen und magischen Buchstabenkombinationen verbunden ist. Höheres gibt sich dem Niederen, Geistiges dem Stofflichen stets durch eine gewisse Transparenz sowie durch das Wort kund, der Unterschied zwischen der Manifestation des Erdgeistes im ersten Teil der «Faust»Dichtung und der Schaffung des Homunculus im zweiten Teil kann nur als graduell gewertet werden - das Prinzip bleibt stets dasselbe. So wie der Mensch zwischen Gott und dem Tier steht, bewegt sich der Homunculus auf dem schmalen Grat, welcher die leblos-amorphe Masse vom beseelten Individuum trennt. Ein diviner Funke, jedoch mit luziferischer Färbung, kennzeichnet die Bahn des Homunculus wie der Schweif den Kometen, und damit ist auch die Erklärung gegeben, weshalb ein solches Monstrum bereits anlagemäßig dem widernatürlichen Untergang geweiht zu sein scheint.

Daß sich fast alle Generationen mit dem Homunculus-Problem befaßten, hat sehr plausible Gründe: Hält doch dieses künstliche Doppelgängerwesen seinem großen Bruder immer den Spiegel vor. So wird es zum Prüfstein für Menschenmögliches, aber auch zum Symbol prometheischer Vermessenheit. Weil sich im Zeitalter der Technik mit ihren Massenvernichtungsmitteln und unzulänglichen Kontrollmöglichkeiten für entfesselte Energien eine Kollektivangst im Unterbewußtsein des Europäers ausbreitete, gewann die Gestalt des Golems seit dem Ersten Weltkrieg erneut an Bedeutung. Denn beide, Mensch und Homunculus, verbindet dadurch, daß sie letztlich von Erde genommen sind und wieder zu Erde werden, ein gemeinsames Schicksal, und wenn es auch in Wirklichkeit nie einen Menschen von Menschenhand gegeben hat, so übertrug das sterbliche Ich alle Sehnsüchte, aber auch alles Grauen vor dem Übersinnlichen auf ihn, gemäß dem Wort aus dem 82.Psalm: "Ihr seid Götter und allzumal Kinder des Höchsten, aber ihr werdet sterben wie Menschen und wie ein Tyrann zugrunde gehen.» 


\section{Schrifttum}

1 Babylonischer Talmud, Band VII/1, Sanhedrin 65b, Berlin/Wien 1925

2 Bцосн Сн. Der Prager Golem, Berlin 1920

3 Eckermann J. P. Gespräche mit Goethe, Leipzig 1955

4. Goethe J.W. v. Sämiliche Werke, Jubiläumsausgabe in 40 Bänden, Stuttgart/Berlin o. J., Band 14: Faust, zweiter Teil

5 Goethe J. W. v. Faust und Urfaust, Wiesbaden 1948

6 Die Kabbala, Das Buch Bahir, Leipzig 1923

8 Die Kabbala, Das Buch Jezira, Ulm 1962

8 Kracauer S. Von Caligari bis Hitler. Ein Beitrag zur Geschichte des deutschen Films, Hamburg 1958

9 Meyrink Gustav, Der Golem, o. O. 1916

10 Offenbach J. Hoffmanns Erzählungen, Textbuch, Stuttgart 1955

Paracelsus, Medizinische, naturwissenschaftliche und philosophische Schriften, Bände I-XIV, herausgegeben von K. SudHoFf, Bände 6-9 München 1922-1925, Bände 1-5 München/Berlin 1928-1933.

11 Paracelsus, Von dem Honig, Band 2, S. 195

12 Paracelsus, Deutsche Originalfragmente zu den fünf Büchern «De vita longa», Band 3, S. 304

13 Paracelsus, Die 9 Bücher «De Natura rerum» (Liber primus), Band 11, S. $316 \mathrm{ff}$.

14. Paracelsus, Liber Azoth, Band 14, S. $549 \mathrm{ff}$.

15 Paracelsus, De pestilitate, Band 14, S. $656 \mathrm{ff}$.

16 Paracelsus, Liber de imaginibus, Band XIII, S. 382

17 Paracelsus, Liber de homunculis, Band 14, S. $325 \mathrm{ff}$.

18 Paracelsus, Liber de animalibus ex Sodomia natis, Band 14, S. $381 \mathrm{ff}$.

19 Peuckert W. E. Paracelsische Zauberei, in: Nova Acta Paracelsica, Band 8, S. 71, Einsiedeln 1957

20 Sсноцем G. Zur Kabbala und ihrer Symbolik, Zürich 1960

21 Sсноцем G. Die jüdische Mystik in ihren Hauptströmungen, Zürich 1957

22 Sterne L. Tristram Shandy, Wiesbaden 1946

23 Zimmermann J. G. Von der Erfahrung in der Arzneykunst, Tom. 1., Zürich 1763

Bibelstellen: 1. Mos. 1, 5; 1, 24; 2, 7. Jesaja 59, 2-5. Psalm 82, 6-7; 139, 16 


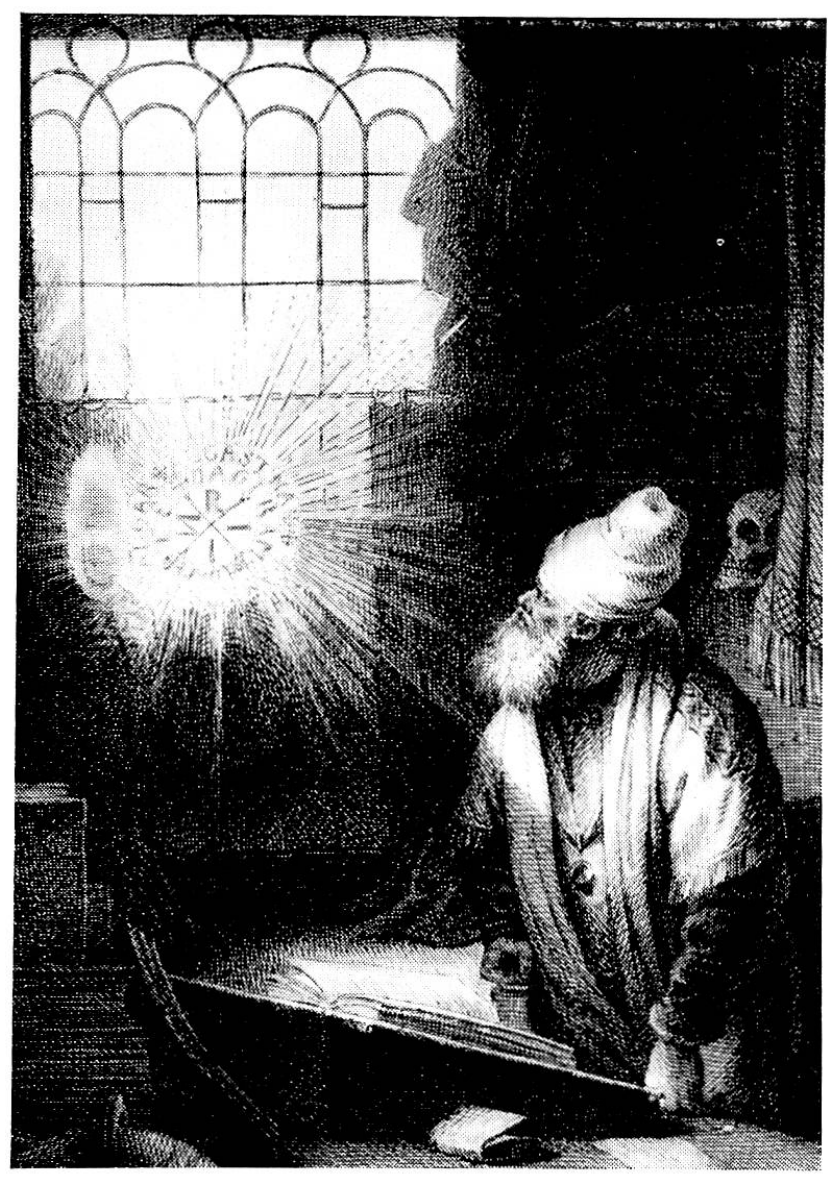

Titelkupfer von H. LIPs zur 1. Ausgabe von GoETHES Faust. Ein Fragment (Göschen, 1790) nach einer Radierung Rembrandts. Transzendentale Phänomene geben sich stets durch Wort (Buchstabenkombinationen), Ton und Licht kund 